\title{
Performance of humidity sensor based on photonic crystal fiber
}

\section{interferometer}

\author{
Suaad Sahib Hindal, Hanan J. Taher
}

Institute of Laser for Postgraduate Studies, University of Baghdad

E-mail: taherhanan@ymail.com

\begin{abstract}
Photonic Crystal Fiber Interferometers (PCFIs) are greatly used for sensing applications. This work presents the fabrication and characterization of a relative humidity sensor based on MachZehnder Interferometer (MZI), which operates in reflection mode. The humidity sensor operation based on the adsorption and desorption of water vapour at the silica-air interface within the PCF. The fabrication of this sensor is simple, it only includes splicing and cleaving the PCF with SMF.PCF (LMA-10) with a certain length spliced to SMF (Corning-28).

The spectrum of PCFI exhibits good sensitivity to humidity variations. The PCFI response is observed for a range of humidity values from $(27 \% \mathrm{RH}$ to $85 \% \mathrm{RH})$, the position of the interference peaks is found to be shifted to longer wavelength with humidity increasing. In this work, a different length of PCFs are used, and the maximum humidity sensitivity of $(5.86 \mathrm{pm} / \% \mathrm{RH})$ is achieved with $(4.5 \mathrm{~cm})$ PCF length, and the rise time of $(8 \mathrm{sec})$ is achieved. This humidity sensor has distinguished features as that it does not require the use of a hygroscopic material, robust, compact size, immunity to electromagnetic interference, and it has potential applications for high humidity environments.
\end{abstract} Key words

Humidity sensors, Optical fiber sensor, Photonic crystal fiber interferometer.

\section{Article info.}

Received: Feb. 2016

Accepted: Mar. 2016

Published: Sep. 2016

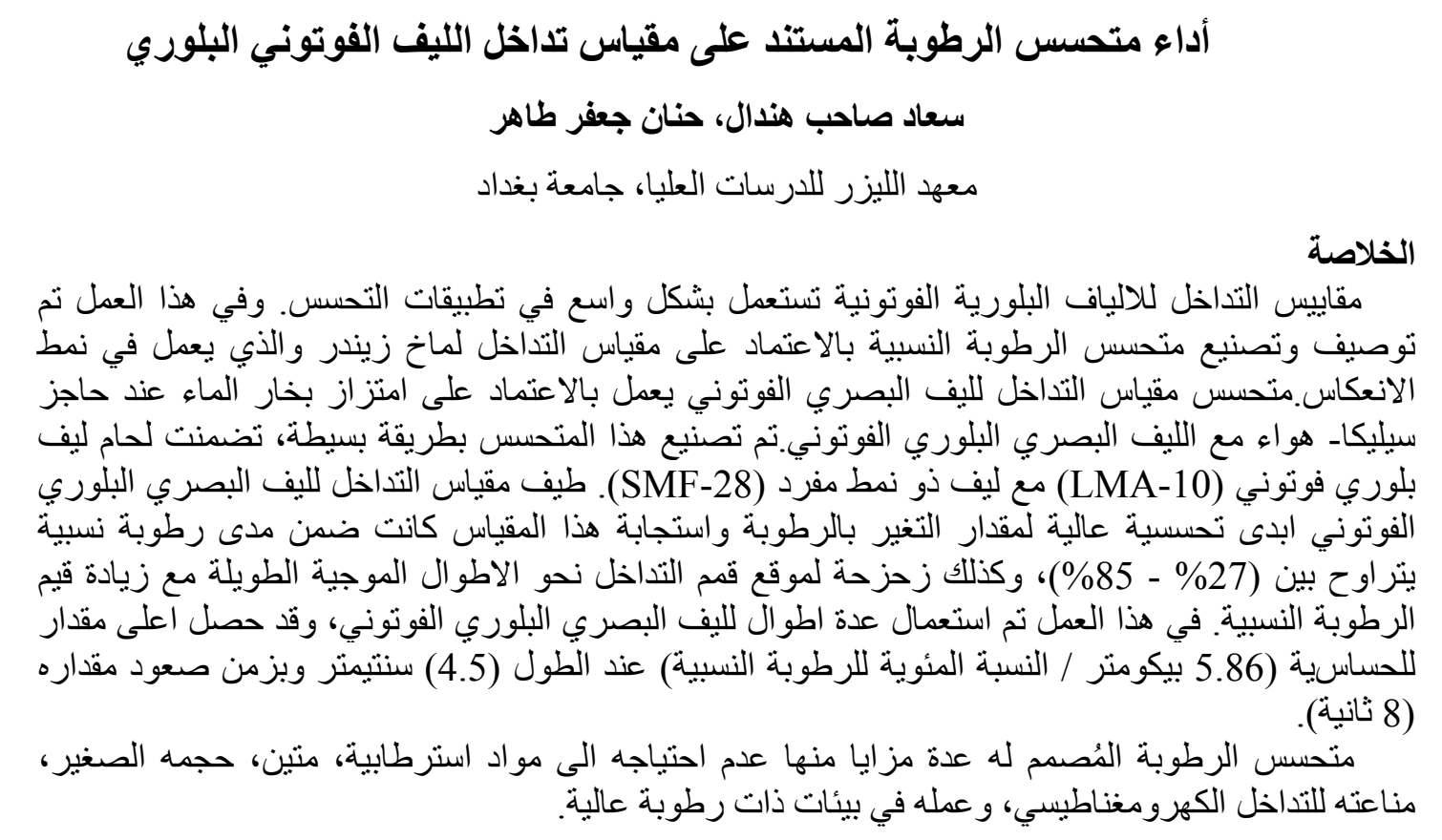




\section{Introduction}

Photonic Crystal Fiber (PCF), which is also called holey fiber or Microstructured Optical Fiber (MOF) appeared in the mid-1990s. PCFs have a periodic layout of microholes that run along the entire length of fiber. They are two types of PCF cross sections: a solid silica core surrounded by air-silica cladding, where the lightguiding mechanism is provided by means of a Modified Total Internal Reflection (MTIR), or a hollow core surrounded by air-silica cladding, where the light-guiding mechanism is based on the Photonic Band Gap (PBG) effect [1].

The presence of air holes in PCF provides a possibility of light propagation in air, or instead of that gives the ability to inject gases/liquids into the air holes. This provides a good controlled interaction between light and matter leading to novel sensing applications that cannot get it with conventional optical fibers $[2,3]$.The applications of PCFs in sensing domains dividing it in two subbranches, depending on the parameter that is measured. These two subbranches are physical (curvature sensor, temperature sensor, vibration sensor, electric and magnetic sensor, and pressure sensor) and biochemical sensors (gas sensor, humidity sensor, $\mathrm{pH}$ sensor, and molecular sensor) [4].

Humidity is an important factor in different fields such as agriculture, food process and storage, chemical, biomedical, weather conditions monitoring, civil engineering, and electronic, etc.[5]. Humidity indicates to the water vapor content in air. It is one of the most measured physical quantities. Humidity measurements can be stated in a variety of terms and units. Relative humidity $(R H)$ is the ratio of the partial pressure of water vapor to the equilibrium vapor pressure of water at the same temperature. It is expressed as a percentage, using the following expression [6]:

$$
R H=\frac{P_{w}}{P_{w s}} \times 100 \%
$$

where $P_{\mathrm{w}}$ is the partial pressure of the water vapor and $P_{\mathrm{ws}}$ is the saturation water vapor pressure. $\mathrm{RH}$ is a relative measurement because it is a function of temperature [6]. Conventional electronic RH sensors are based on monitoring the changes in electrical capacitance or conductivity, and because of the electrical leakage, especially in a high humidity environment, this types of sensors have the disadvantage of inaccuracy. So, the optical fiber based RH sensors (compared with electronic RH sensors) offer many advantages, such as small size, low weight, immunity to electromagnetic interference, and remote monitoring. There are a wide range of $\mathrm{RH}$ sensing techniques based on optical fibers, including long period gratings (LPG), fiber Bragg gratings, plastic optical fibers, surface plasmon resonance, and tapered optical fibers[7].

Relative Humidity (RH) sensor based on a PCFI has been submitted in this paper, which have an unique features such as it doesn't require any hygroscopic material to measure humidity and the sensor tip is made of single material (silica). Because of these features this sensor is suitable for working in harsh and high-temperature environments. For an interferometric type fiber optic RH sensor, the sensing mechanism relies on the perturbation of the light signal phase properties that travelling in the optical fiber introduced by the humidity change. The phase change detection is realized by mixing the signal of interest with a reference signal, then converting the phase difference into wavelength shift or an optical intensity change [6]. In this paper, the sensing element is just a stub of PCF spliced to SMF, this forms 
a reflection-type PCFI, and the spectrum of this sensor exhibits good sensitivity to humidity variations.

\section{Experimental}

Humidity sensor based on reflection type of the PCFI has been proposed. First, the coating of a stub of PCF (LMA-10) and conventional optical fiber (Corning, SMF-28) are removed by using a mechanical stripping. Then, the second step is cleaving the PCF and SMF, which is done by fiber cleaver, and third step is cleaning the fibers. Then, the stub of PCF (LMA$10)$ is spliced to conventional optical fiber (Corning, SMF-28) by a conventional splicing machine. The
PCF (LMA-10) designed for an endless single-mode operation is used, it has four layers of air holes arranged in a hexagonal pattern around a solid silica core, the fiber has a core size diameter of $10 \mu \mathrm{m}$, voids with a diameter of $3.1 \mu \mathrm{m}$, pitch of $6.6 \mu \mathrm{m}$ and outer diameter of $125 \mu \mathrm{m}$. These dimensions of the PCF (LMA-10) alignment and splicing with the SMF with a splicing machine, and due to mode-field diameter (MFD) mismatch compared to other PCFs, the loss was minimize. During the splicing process and due to surface tension, the voids of the PCF collapse within a microscopic region $(\sim 300 \mu \mathrm{m})$ near the splice point, as shown in the Fig. 1.

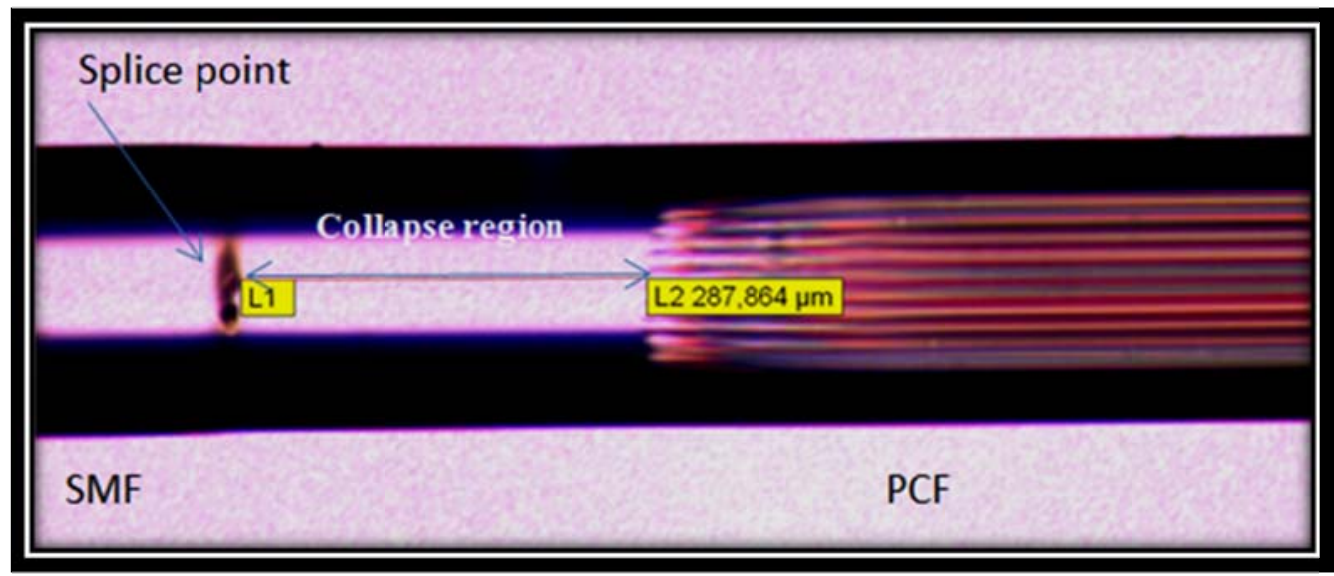

Fig. 1: Microscope image of the splice zone between PCF (LMA-I0) and the SMF.

After the splicing, the PCF is cleaved with a standard cleaving machine so that the end of the PCF acts as a reflecting surface (Mirror). The PCF holes are left open to the ambient atmosphere. The light guidance mechanism in PCF is by modified total internal reflection (MTIR).

The working principle of the PCFI is relied on the excitation and recombination of modes occurring in the zone of the PCF in which the voids of the PCF are collapsed. The fundamental SMF mode diffract when it enters the collapsed region of the
PCF. So, excitation of two core modes in the PCF occurs, because of diffraction the mode broadens. Then modes transfer through the PCF until they reach the PCF cleaved end from where they are reflected. Then, the reflected modes are recombined as a single SMF core mode, when they reenter the collapsed region [8, 9]. Fig. 2 shows the setup of the humidity sensor based on PCFI, light source (1550nm) is launched into the interferometer through the Fiber Optic Circulator (FOC), and light that reflected from the cleaved end is fed to the Optical Spectrum Analyzer (OSA). 


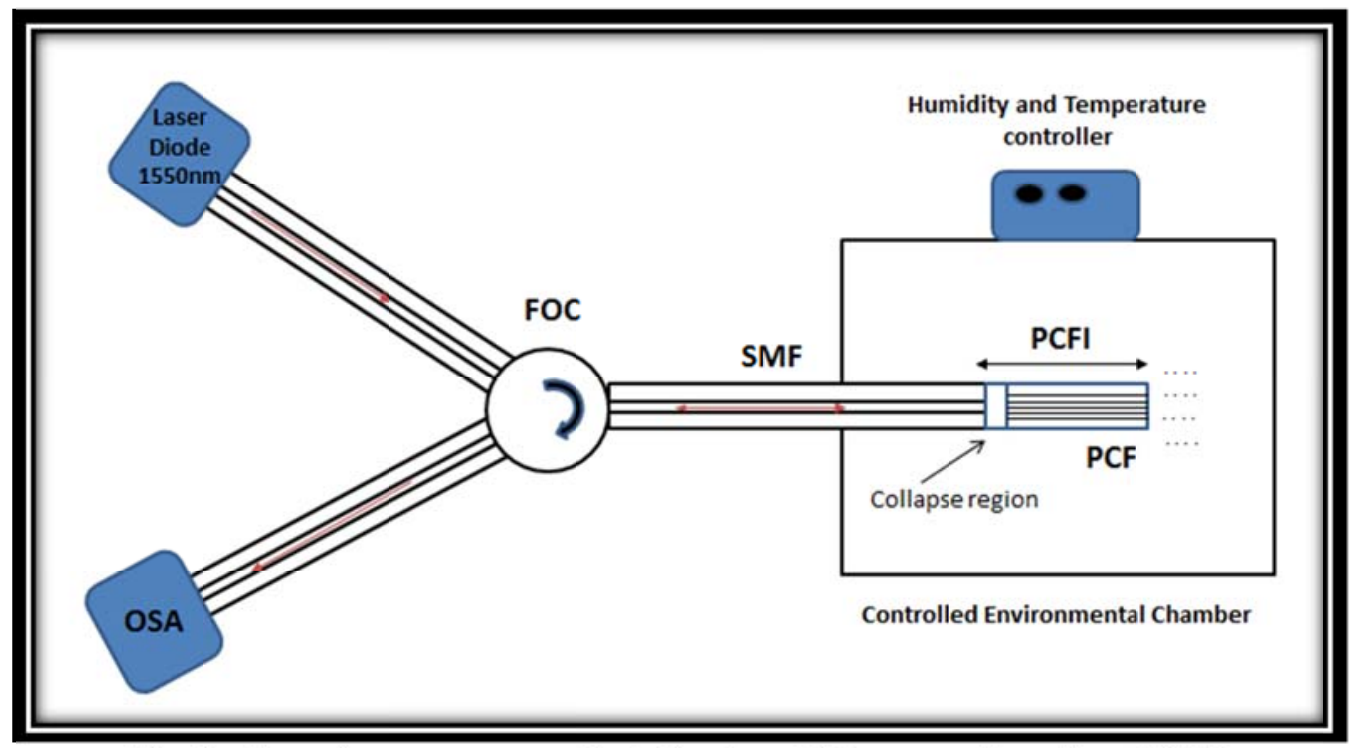

Fig. 2: Experimental setup of relative hamidity sensor based on PCFI.

In this setup, the shift of the interference peaks is tracking with high resolution. A different lengths of the PCF section used to show the sensitivity dependence on PCF length.

The fabricated sensor response to humidity variations is studied at room temperature and normal atmospheric pressure by putting it in an environmental chamber, which is a cuboid-shape sealed chamber, fabricated from Polyvinyl chloride (PVC) plastic. It consists of dry/wet air flow system that can vary the internal humidity in the chamber $(27 \% \mathrm{RH}$ $85 \% \mathrm{RH}$ ), there are three fans (the first fan is pumps a dry air form container containing a silica gel, the second fan pumps a wet air from container containing distilled water and heater (70watt), and the last fan is in the surface of chamber to expel the air). A calibrated electronic humidity (XMT9007-8 temperature \& humidity control instrument) is used for monitoring the humidity and temperature inside the chamber.

\section{Results and discussion}

The response of the PCFI is observed for a range of humidity values $(27 \%, 30 \%, 40 \%, 50 \%, 60 \%$, $70 \%, 80 \%$ and $85 \%) \mathrm{RH}$. The position of the interference peak is found shifted within the humidity variations to the longer wavelength (red shift). The sensitivity of the sensor is calculated by dividing the experimentally measured PCFI response to the relative humidity. The Relative Humidity $(\mathrm{RH})$ response of the PCFI device is studied with different PCF lengths and different ambient relative humidity values (at room temperature and normal atmospheric pressure).

Figs. 3 and 4, show the shifting of the interference peak of the reflected light from PCFI for the submitted sensors for different lengths of PCF and with $4.5 \mathrm{~cm}$ PCF length, respectively. 


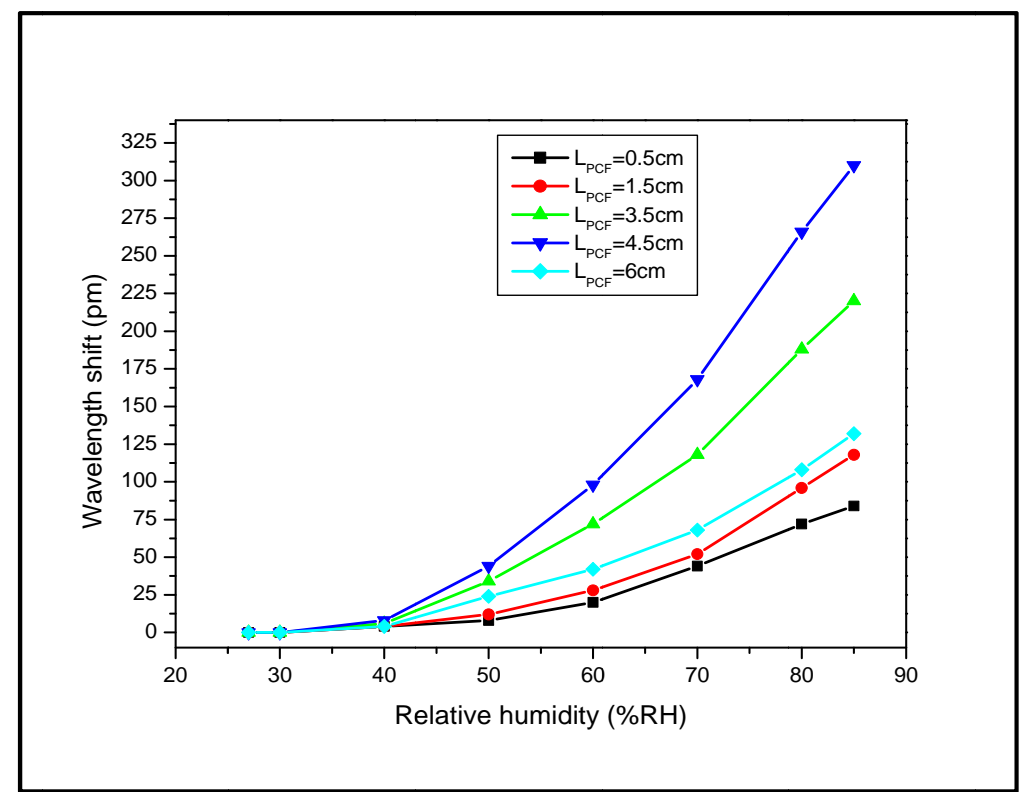

Fig. 3: Interference peaks shift of PCFI with respect to relative humidity and for different lengths of PCF.

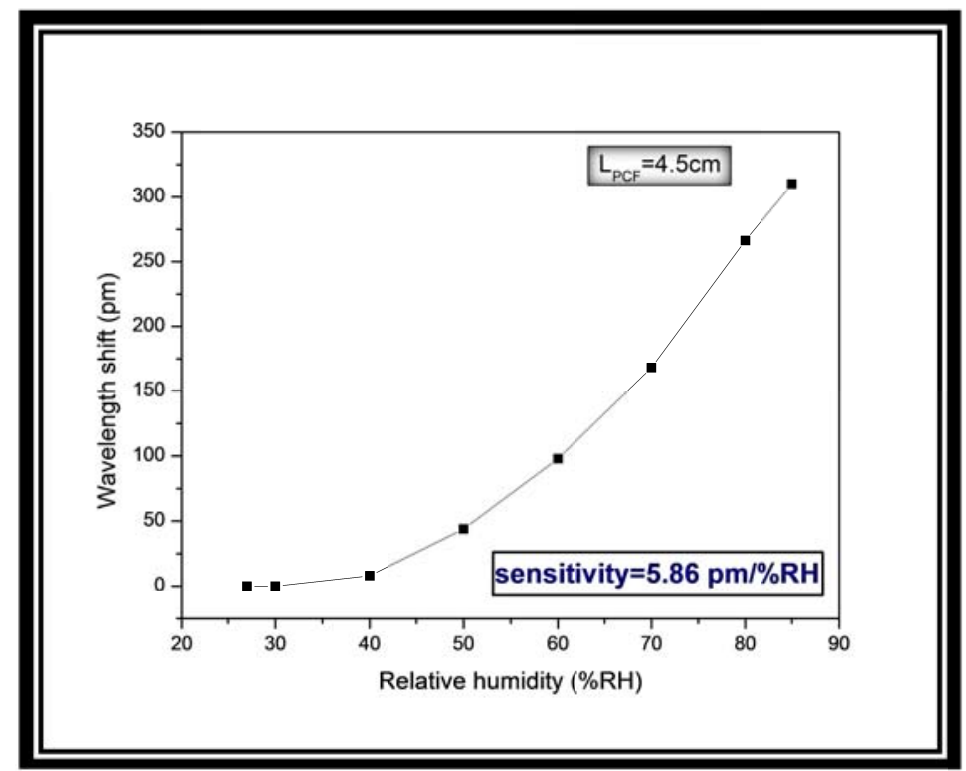

Fig. 4: Interference peaks shift of PCFI with respect to relative humidity, with $4.5 \mathrm{~cm}$ PCF length.

From the previous figures, it is observed that there is no shifting in interference peak between $27 \%-40 \%$ $\mathrm{RH}$ region for all PCF lengths, the shift being to appear for relative humidity value $40 \% \mathrm{RH}$. This is because water has a hydrogen-bonded network (icelike), which grows up as the relative humidity increases from $0 \%$ to $30 \%$. The liquid water structure starts appearing in the $\mathrm{RH}$ range of $30-60 \%$, while the structure of ice-like continues growing to saturation [10].

The sensitivity in $\mathrm{pm} / \% \mathrm{RH}$ calculated from the linear fitting of relative humidity versus wavelength curve. It is observed that PCFI with length $(4.5 \mathrm{~cm})$ shows the higher sensitivity (5.86 pm /\%RH) compared with the other PCFI lengths. Fig.5 shows the relation between the PCF lengths and the sensitivity of the device. 


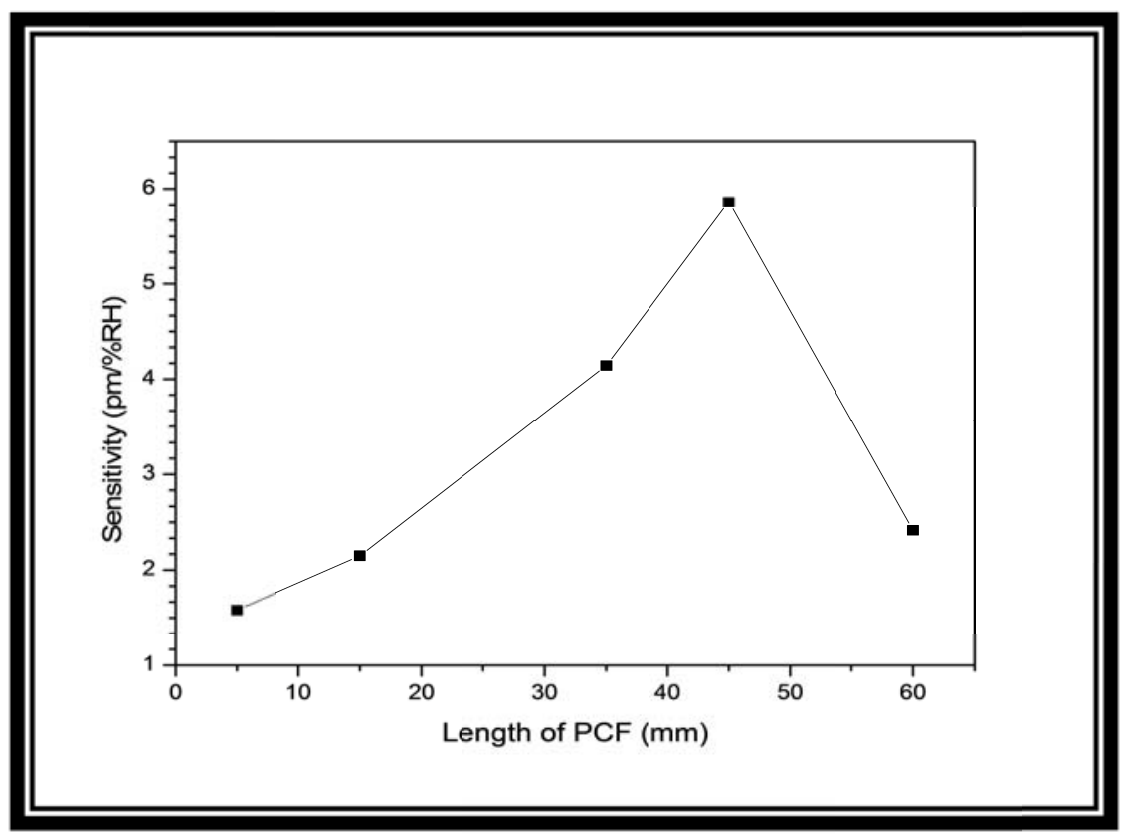

Fig. 5: The relationship between of PCF lengths and sensitivity of proposed sensor.

When the length of the device decreases, the sensitivity of the PCFI to the relative humidity was decreasing, as shown in the Fig. 5. The reason of this behaviour is due to the fact that for a small PCF length, the available length for interaction between the adsorbed water vapor with the cladding mode is smaller which leads to that phase difference obtained between the interfering modes will be smaller.

For much longer PCF length $(6 \mathrm{~cm})$, the sensitivity of the device will be decrease; this behaviour is due to the fact that in the longer PCFI the infiltration of water molecules may need a long time. Also, the fringe visibility will diminish when the length of the PCF increasing which leads to increase the propagation loss of the interfering cladding mode.
Furthermore, the fringe spacing for a longer PCFI will be narrow which limits the measurement range of the PCFI [6].

To calculate the rise time of the sensor, PCFI (with specific length $4.5 \mathrm{~cm}$ which is the length that shows the higher sensitivity to relative humidity variations) is exposed to an environment with rapid changes of the RH. First, keep the RH in the chamber at $40 \% \mathrm{RH}$, and then rapidly increase the humidity of the chamber to $85 \%$ (at room temperature and normal atmospheric pressure). The measured rise time of the sensor is shown in Fig.6, the sensor has a fast response to humidity variations and the estimated response time (from $10 \%$ to $90 \%$ of the signal maximum) is about $8 \mathrm{sec}$ when the $\mathrm{RH}$ changes from $40 \%$ to $85 \%$ at wavelength $=1550 \mathrm{~nm}$. 


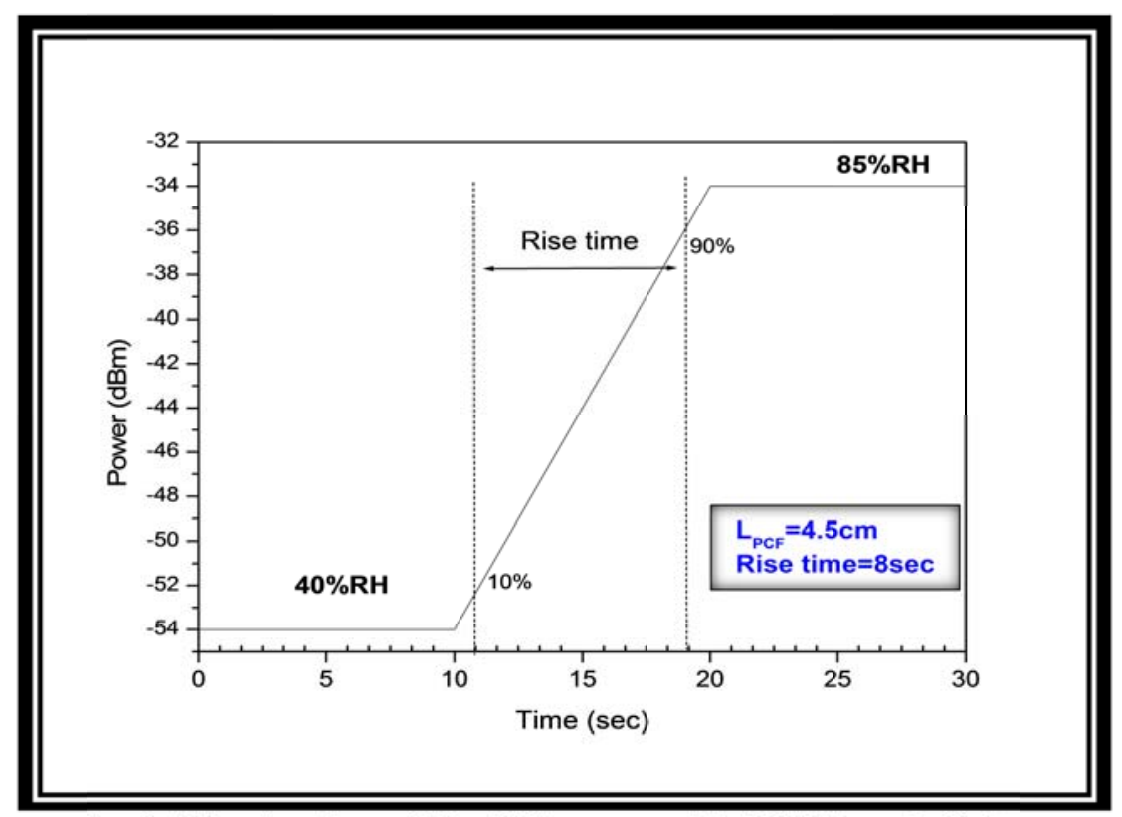

Fig. 6: The rise time of the $R H$ sensor with PCF length (4.5crn).

\section{Conclusions}

Mach-zehnder interferometer (MZI) for humidity detection based on PCF is demonstrated; this PCFI is simple and operates in reflection mode. Its fabrication involves only splicing a short pieces of one end of PCF (LMA10) with SMF (Corning-28), and cleave the free end of PCF to act as a mirror, and this sensor does not require the use of any hygroscopic material.

The sensor sensitivity of the RH depends on the PCF length, and the sensor with $4.5 \mathrm{~cm}$ PCF length is more sensitive to RH variations, but for much longer PCF length the sensitivity decrease. The maximum sensitivity of the sensor is $5.86 \mathrm{pm} / \% \mathrm{RH}$ in the $\mathrm{RH}$ ranges of $40-85 \%$, and with $4.5 \mathrm{~cm}$ PCF length, and the minimum sensitivity of the sensor is $1.58 \mathrm{pm} / \% \mathrm{RH}$ with $0.5 \mathrm{~cm}$ PCF length.

This sensor has a fast response to humidity variations, the rise time when the $\mathrm{RH}$ changes from $40 \%$ to $85 \%$ for this sensor with PCF length $4.5 \mathrm{~cm}$ and at wavelength $1550 \mathrm{~nm}$ is equal $8 \mathrm{sec}$.

\section{References}

[1] A. Massaro, "Photonic Crystals Introduction, Applications and Theory", Chapter 8, In Tech, 2012.
[2] O. Frazao, J. Santos, F. Araujo, L. Ferreira, Laser \& Photon. Rev.2, 6 (2008) 449-459.

[3] J. Chen, "Nanochemistry and Sensing in Photonic Crystal Fibers", Max-Planck Institute for the Science of Light, University of ErlangenNuremberg 2010.

[4] A. Pinto, A. Lopez-Amo, Journal of Sensors, 12 (2012) 1-21.

[5] L. Alwis, T. Sun, K. Grattan, Measurement, 46 (2013) 4052-5074.

[6] J. Mathew, "Development of Novel Fiber Optic Humidity, Sensors and Their Derived Applications", Dublin Institute of Technology, 2013.

[7] Q. Wu, Y. Semenova, J. Mathew, P. Wang, G. Farrell, Opitics Letters, 36, 10 (2011) 1752-1754.

[8] J. Villatoro, V. Minkovich, V. Pruneri, G. Badenes, Optics Express, 15, 4 (2007) 1491-1496.

[9] J. Villatoro, M. Kreuzer, R. Jha, V. Minkovich, V. Finazzi, G. Badenes, V. Pruneri, Optics Express, 17 (2009) 1447-1453.

[10] B. David, H. Seong, J. Phys. Chem. B, 109, 35 (2005) 1676016763. 